\title{
Thick as Thieves? Dishonest Behavior and Egocentric Social Networks
}

\section{Citation}

Lee, Jooa Julia, Dong-Kyun Im, Bidhan Parmar, and Francesca Gino. "Thick as Thieves? Dishonest Behavior and Egocentric Social Networks." Harvard Business School Working Paper, No. 15-064, February 2015.

\section{Permanent link}

http://nrs.harvard.edu/urn-3:HUL.InstRepos:13994837

\section{Terms of Use}

This article was downloaded from Harvard University's DASH repository, and is made available under the terms and conditions applicable to Open Access Policy Articles, as set forth at http:// nrs.harvard.edu/urn-3:HUL.InstRepos:dash.current.terms-of-use\#OAP

\section{Share Your Story}

The Harvard community has made this article openly available.

Please share how this access benefits you. Submit a story.

Accessibility 


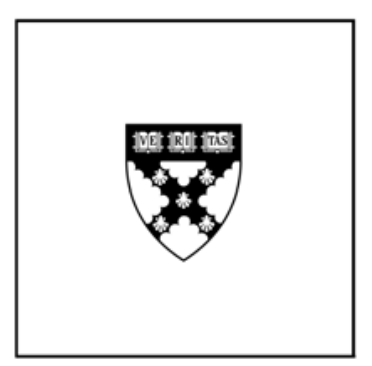

\title{
Thick as Thieves? Dishonest Behavior and Egocentric Social Networks
}

\author{
Jooa Julia Lee \\ Dong-Kyun Im \\ Bidhan Parmar \\ Francesca Gino
}

\section{Working Paper}

15-064

February 10, 2015 
Running Head: CHEATER'S NETWORK

Thick as Thieves?

\title{
Dishonest Behavior and Egocentric Social Networks
}

\author{
Jooa Julia Lee ${ }^{1}$ \\ Dong-Kyun $\mathrm{Im}^{2}$ \\ Bidhan Parmar ${ }^{3}$ \\ Francesca Gino ${ }^{1}$ \\ ${ }^{1}$ Harvard University \\ ${ }^{2}$ University of Seoul \\ ${ }^{3}$ University of Virginia
}

\begin{abstract}
Author Note
We greatly appreciate the support of the Harvard Business School and Computer Lab for Experimental Research (CLER). We are also grateful to Annie Giebelhaus, Ceylan Oymak, and Yian Xu for their assistance.
\end{abstract}




\begin{abstract}
People experience a threat to their moral self-concept in the face of discrepancies between their moral values and their unethical behavior. We theorize that people's need to restore their view of themselves as moral activates thoughts of a high-density personal social network. Such thoughts also lead people to be more likely to engage in further unethical behavior. In five experiments, participants reflected on their past unethical behavior, and then completed a task designed to measure network density. Those who cheated more frequently in the past, recalled their negative moral identity, or decided to lie were more likely to activate a high-density network (Experiment 1-3). Using a mediation-by-moderation approach (Experiment 4), we confirm that this link between dishonesty and network density is explained by a threat to positive self-concept. Importantly, activating a dense network after engaging in dishonest behavior allows further dishonest behavior in a subsequent task (Experiment 5).
\end{abstract}

Keywords: social network structure; network density; dishonesty; ethics; self-affirmation 


\section{Thick as Thieves? Dishonest Behavior and Egocentric Social Networks}

Beyond its obvious financial consequences, dishonest behavior, when detected, can trigger distrust and negative emotions, and is therefore costly for social and romantic relationships (McCornack \& Levine, 1990; Miller, Mongeau, \& Sleight, 1986). Previous research has found that dishonest behavior can even have negative consequences when it goes unnoticed. Given the widespread need to view oneself as honest (Mazar, Amir, \& Ariely, 2008) and maintain a moral self-image (Monin \& Jordan, 2009), ethically questionable behavior can create significant discomfort, as it highlights the discrepancies between one's moral self-concept and actual behavior (E. Aronson, 1968; 1969; Higgins, 1987). As a result, individuals may experience reduced self-esteem or moral emotions such as guilt or shame after engaging in dishonest behavior (Klass, 1978).

To reduce the discomfort of violating moral norms, people become motivated to seek out opportunities to salvage their moral self-concept. They may alleviate a sense of threat to their moral self-concept by relaxing their moral norms through moral disengagement and moral forgetting (Bandura, 1990; Shu \& Gino, 2012; Shu, Gino, \& Bazerman, 2011). Motivated to normalize their deceptive behavior in an effort to protect their moral self-concept (Sagarin, L Rhoads, \& Cialdini, 1998), liars also tend to perceive the recipient of their lies as less honest than they would otherwise. Alternatively, they may seek moral redemption by complying with requests to help others (Carlsmith \& Gross, 1969; McMillen, 1971; McMillen \& Austin, 1971) or engaging in other types of prosocial and ethical behavior (Cialdini, Darby, \& Vincent, 1973; J. Jordan, Mullen, \& Murnighan, 2011).

The common finding in this body of research that dishonest behavior poses a threat to one's moral self-concept and triggers protective and compensatory behavior raises the possibility 
that one's dishonest behavior may activate thoughts of a densely structured social network as a way of restoring positive self-concept. In sociology, the traditional conception of community as spatially defined has shifted to the consideration of relationally defined communities and the networks built around the self (Chua, Madej, \& Wellman, 2011). The concept of the ego network, or "personal community," has become increasingly important. Among the various networks that can be built around oneself, a high-density network of tightly connected "alter egos" provides several psychological benefits for ego protection. Network density, an indicator of the extent to which a network is closely knit, is theorized to enhance group cohesion and intra-group bonds (Barnes, 1969; Blau, 1977) and to facilitate communication and knowledge transfer across social network (Reagans \& McEvily, 2003). Due to these characteristics, high-density networks help establish shared norms and trusting relationships (Coleman, 1988), and reduce loneliness (Stokes, 1985). In addition, being part of cohesive network improves emotional adjustment. For example, education research has shown that students who belong to cohesive groups tend to experience less anxiety and performance stress than those who do not (Bowers, Weaver, \& Morgan, 1996; M. E. Shaw \& Shaw, 1962). In the aftermath of dishonest behavior, individuals may think about those closest to them to restore their threatened self-concept.

If a high-density network buffers a threat to one's moral self-concept, then would this lead to further dishonest behavior in a subsequent task? It could be argued that a high-density network contributes to perpetuating one's dishonest behavior. For example, a dense network could lead to rapid dissemination of unethical behavior (Brass, Butterfield, \& Skaggs, 1998). In fact, under performance pressure, close-knit networks fueled unethical practices among medical professionals, and trust among individuals in such networks functioned as a medium to conceal unethical practices (Türker \& Altuntaş, 2014). Just as dishonest behavior increases distrust 
toward the deceived (Sagarin et al., 1998), a dense and cohesive network may also be conducive to inter-group biases (Labianca, Brass, \& Gray, 1998), thus providing further group-based justifications for unethical behavior. Indeed, in one study, belonging to a cohesive group helped in-group members rationalize their prejudice against out-group members (Effron \& Knowles, 2015).

Drawing from a theory that frames unethical behavior as a primarily social phenomenon (Brass et al., 1998), we explore the consequences of unethical behavior in cognitive activation of the egocentric social network. We hypothesize that thinking about or directly engaging in dishonest behavior would activate a high-density network, measured as the extent to which network members identified by participants know one another. We also propose that selfaffirmation will buffer the unintended effects of unethical behavior on the activation of highdensity networks by sustaining a person's sense of moral adequacy (Steele, 1988). Lastly, we test whether triggering thoughts of a high-density network as a response to one's dishonest behavior has positive or negative consequences for subsequent moral behavior.

We tested our main hypotheses in five experiments. Together, our findings provide novel empirical evidence that dishonest behavior leads to the cognitive activation of a dense social network as a defensive response to a threat to one's moral self-concept. They also show that triggering a high-density network as a response to one's dishonest behavior has negative consequences in subsequent moral behavior.

\section{Experiment 1: Cheaters Activate a High-density Network}

In this study, we test our hypothesis that individuals who report having cheated or lied more frequently will be more likely to activate a high-density network. A high-density network is measured by the extent to which the network members identified by participants know one 
another. We first asked participants to rate the frequency of their own unethical behavior and then to report on their social networks.

\section{Method}

Participants. One hundred ninety-eight individuals $\left(M_{\text {age }}=23.02, S D_{a g e}=4.07 ; 51 \%\right.$ male $)$ from the Boston/Cambridge area participated in this study as part of a laboratory study that aimed to recruit approximately 200-250 participants. Participants completed a 15-minute survey that was part of a one-hour long series of experiments they completed at individual computer terminals. They received $\$ 20$ for completing the study and were debriefed shortly after they finished the survey.

Frequency of ethically questionable behavior. We asked participants how frequently they have engaged in particular ethically questionable behaviors (Barkan, 2007; adapted from Gino, Norton, \& Ariely, 2010). The 13-item scale included statement such as "be in the express line with too many groceries," and lying "Sorry I am late, traffic was terrible" (from 1=never to $5=$ all of the time, and 6=not applicable; See Appendix A for the full description of the scale items). We coded the items to which participants responded "not applicable" as missing and created a summary variable based on items for which participants provided responses between 1 and $5(\alpha=0.85)$.

Cognitive activation of network. To measure individuals' social networks and social capital, sociologists have examined individuals' core discussion network (Burt, 1984; Marsden, 1987; McPherson, Smith-Lovin, \& Brashears, 2009; Small, Deeds Pamphile, \& McMahan, 2015), typically using the "name generator module," which asks survey respondents to list the set of individuals they regularly turn to when discussing important matters. Adapting from these past studies, we gave participants the following instructions: "From time to time, most people discuss 
important matters with other people. Looking back over the last six months, who are the people with whom you discussed matters important to you? Please write down up to ten names using the initials." Once participants write down up to ten persons' initials in a survey programmed in Qualtrics.com, we presented all possible pairs of the contacts generated by the participants (up to 45 possible pairs, if a participant generated all 10 contacts). ${ }^{1}$ Participants indicated the strength of relations for each pair, from $1=$ no relationship to $7=$ extremely strong relationship. We dichotomized this variable to indicate whether the particular pair of contacts know each other or not, and formed a measure of network size (a total number of contacts generated; $M=8.37$, $S D=2.61)$. Following the procedures used in Scott (1991) to measure cognitive activation of network structure, network density was calculated by dividing the total number of network ties (i.e., network size) by the total number of possible ties (Scott, 1991).

Demographics. We also collected demographic information, including age, gender, level of education (1=high school, 2=some college, 3=associate's degree, 4=bachelor's degree, 5=master's degree, $6=$ doctorate or professional degree), monthly household income (1=none, $2=$ under $\$ 60,3=\$ 60-499,4=\$ 500-999,5=\$ 1,000-\$ 1,999,6=\$ 2,000-2,999,7=\$ 3,000-3,999$, $8=\$ 4,000-4,999,9=\$ 5,000-7,499,10=\$ 7,500-9,999,11=$ over $\$ 10,000$ ), and religiosity (from $1=$ not at all religious to $5=$ very religious).

\section{Results}

Table 1 shows the descriptive statistics of the main variables and their zero-order correlations. Using hierarchical multiple regression analyses, we found that the self-reported frequency of ethically questionable behavior is positively correlated with the activation of a more dense network (Model 1 in Table 2). In Model 2 and 3, we show that this relationship between

\footnotetext{
${ }^{1}$ The total number of possible pairs is calculated by $\mathrm{N}(\mathrm{N}-1) / 2$, where $\mathrm{N}$ is the total number of contacts.
} 
ethically questionable behavior and network density is robust after controlling for network size and demographics.

Table 1

Descriptive Statistics and Zero-order Correlations for Experiment 1

\begin{tabular}{|c|c|c|c|c|c|c|c|c|}
\hline & Mean (SD) & 1 & 2 & 3 & 4 & 5 & 6 & 7 \\
\hline 1. Ethically questionnable behavior & $1.95(0.60)$ & & & & & & & \\
\hline 2. Network size & $7.65(2.85)$ & 0.13 & & & & & & \\
\hline 3. Network density & $0.70(0.19)$ & $0.19 * *$ & $-0.34 * * *$ & & & & & \\
\hline 4. Age & $23.06(4.08)$ & -0.10 & $-0.14 *$ & 0.01 & & & & \\
\hline 5. Female & $1.48(0.50)$ & -0.04 & 0.01 & -0.02 & 0.07 & & & \\
\hline 6. Education & $2.75(1.28)$ & -0.09 & -0.01 & 0.01 & $0.59 * * *$ & $0.16^{*}$ & & \\
\hline 7. Income & $6.68(3.86)$ & 0.01 & -0.02 & 0.06 & -0.02 & 0.03 & 0.10 & \\
\hline 8. Religiosity & $2.48(1.28)$ & 0.05 & 0.08 & -0.02 & -0.00 & -0.06 & -0.02 & -0.03 \\
\hline
\end{tabular}

Note: ${ }^{*} p<0.05,{ }^{* *} p<0.01, * * * p<0.001$

Table 2

Results of Hierarchical Multiple Regression Analyses for Experiment 1

\begin{tabular}{|c|c|c|c|c|c|c|c|c|c|c|}
\hline & \multirow{3}{*}{ Predictor Variables } & \multicolumn{9}{|c|}{ Network Density } \\
\hline & & \multicolumn{3}{|c|}{ Step 1} & \multicolumn{3}{|c|}{ Step 2} & \multicolumn{3}{|c|}{ Step 3} \\
\hline & & $\mathrm{b}$ & $\mathrm{SE}$ & $p$ & $\mathrm{~b}$ & $\mathrm{SE}$ & $p$ & $\mathrm{~b}$ & SE & $p$ \\
\hline 1 & Ethically questionable behavior & 0.06 & 0.02 & .006 & 0.07 & 0.02 & .000 & 0.07 & 0.02 & .001 \\
\hline 2 & Network size & & & & -0.03 & 0.00 & .000 & -0.03 & 0.00 & .000 \\
\hline \multirow[t]{5}{*}{3} & Age & & & & & & & -0.00 & 0.00 & .90 \\
\hline & Female & & & & & & & -0.00 & 0.02 & .83 \\
\hline & Education & & & & & & & 0.00 & 0.01 & .77 \\
\hline & Income & & & & & & & 0.00 & 0.00 & .55 \\
\hline & Religiosity & & & & & & & 0.00 & 0.01 & .79 \\
\hline & $\mathrm{N}$ & \multicolumn{3}{|c|}{192} & \multicolumn{3}{|c|}{192} & \multicolumn{3}{|c|}{192} \\
\hline & Overall R-squared & \multicolumn{3}{|c|}{0.04} & \multicolumn{3}{|c|}{0.17} & \multicolumn{3}{|c|}{0.17} \\
\hline & Overall F & \multicolumn{3}{|c|}{7.77} & \multicolumn{3}{|c|}{19.72} & \multicolumn{3}{|c|}{5.59} \\
\hline & Change in R-squared & & & & \multicolumn{3}{|c|}{0.13} & \multicolumn{3}{|c|}{0.00} \\
\hline & Change in $\mathrm{F}$ & & & & \multicolumn{3}{|c|}{$30.47 * * *$} & \multicolumn{3}{|c|}{0.11} \\
\hline
\end{tabular}

Note: ${ }^{*} p<0.05, * * p<0.01, * * * p<0.001$

\section{Discussion}


In an initial correlational study, we found that participants who reported more frequent unethical behavior tended to trigger a more dense social network than those who reported less frequent unethical behavior.

\section{Experiment 2: Liars Activate a High-density Network}

In Experiment 2, we show that not only self-reported frequency of ethically questionable behavior but also a decision to lie in the task is positively associated with activating more dense networks. We first asked participants to decide whether or not to lie to earn more money in a game and then to report on their social networks.

\section{Method}

Participants. We predetermined 160 to be the sample size to give this study adequate power $(1-\beta>0.80)$ to detect a medium-sized effect $(r=0.30)$. However, six participants reported having technical problems with the name generator and were thus removed. As a result, a total of 154 individuals $\left(M_{a g e}=34.05, S D_{\text {age }}=10.72 ; 54 \%\right.$ male $)$ participated in a 15 -minute online study through Amazon's Mechanical Turk and received \$0.50 participation fees with an opportunity to earn additional $\$ 0.50$ depending on their decision in the game.

Procedure. We asked participants to play a game (Gneezy, 2005) which requires them to decide whether to lie to another participant to earn a $\$ 0.50$ bonus. In this game, all participants were led to believe that they were paired randomly with another anonymous player (Player 2). In fact, all were assigned to the role of Player 1. The participants were given information about two possible monetary payoffs that they were told Player 2 would not be aware of: (1) Option A, which would give $\$ 0.50$ to Player 1 and $\$ 0.00$ to Player 2, and (2) Option B, which would give $\$ 0.00$ to Player 1 and $\$ 0.50$ to Player 2 . They were then asked to send one of two messages to Player 2: a truthful message (“Option B will earn Player 2 more money than Option A”) or a lie 
(“Option A will earn Player 2 more money than Option B”). We used this decision as a measure of an individual's willingness to lie to benefit oneself.

We then repeated the same procedure used in Experiment 1 to measure participants' cognitive activation of their social networks, followed by the demographic questionnaire.

\section{Results}

As hypothesized, individuals who decided to lie to their counterpart $(M=0.79, S D=0.27)$ activated a more dense network than did those who decided not to lie $(M=0.69, S D=0.29)$, $t(152)=-2.21, p=.03, \mathrm{CI}=[-0.19,-0.01], d=-0.36$. As in Experiment 1, the relationship between one's decision to lie and network constraint did not change significantly after adding the network size and demographics variables (age and gender) as covariates, $\mathrm{B}=0.11, S E=0.04, p=.014$.

\section{Discussion}

Along with Experiment 1 which provide self-reported data, these behavioral results provide additional support for our hypothesis that one's decision to engage in unethical behavior predicts triggering a denser network.

\section{Experiment 3: Recalling a Dishonest Self Activates a High-density Network}

In Experiment 1 and 2, we show a positive correlation between one's lack of morality (both self-reported and behavioral) and network density. To address the problem of reverse causality, in Experiment 3, we experimentally manipulated one's moral self-concept. We test the hypothesis that it is the negative moral self-concept that triggers one's high-density network, but not the positive moral self-concept.

\section{Method}

Participants. We pre-determined 160 to be the target sample size such that this study has adequate power $(1-\beta>0.80)$ to detect a medium-sized effect $(f=0.30)$. We stopped data collection 
when we had a total of 160 individuals, but excluded 17 participants who did not follow instructions. A total of 143 individuals $\left(M_{a g e}=39.91, S D_{a g e}=13.49 ; 36 \%\right.$ male $)$ participated in a 20-minute online study through Amazon’s Mechanical Turk and received $\$ 0.50$ participation fees.

Design and task. Participants were randomly assigned to one of the three conditions: negative moral identity, positive moral identity, and a control condition. We adapted methods that have been shown to influence one's moral identity (Reed, Aquino, \& Levy, 2007; Sachdeva, Iliev, \& Medin, 2009). In the negative moral identity condition, participants received a list of five negative moral traits: disloyal, greedy, mean, dishonest, and selfish. In the positive moral identity condition, they received a list of positive moral traits: caring, generous, fair, honest, and kind. Participants in the control condition received a list inanimate objects: books, keys, house, desk, and letter. All participants were then asked to write a short story about themselves using the words they received. An example of the stories provided in each condition is provided in Table 3.

We used the same methods as in Experiment 1 and 2 to elicit network size and density, followed by a demographics survey.

Table 3

Example Stories in Each Experimental Condition for Experiment 3

\begin{tabular}{|c|c|c|}
\hline Negative Moral Identity & Positive Moral Identity & Control \\
\hline $\begin{array}{l}\text { "There was a time in my life } \\
\text { when I was a selfish person, } \\
\text { someone I would not want to be } \\
\text { friends with now. One night after } \\
\text { everyone had left, I stole a } \\
\text { present from under the office } \\
\text { Christmas tree that belonged to } \\
\text { another employee. It was a mean } \\
\text { thing to do, and showed just how }\end{array}$ & $\begin{array}{l}\text { "One of the best times of my life } \\
\text { was when I found what appeared } \\
\text { to be an honest man living under } \\
\text { a bridge. I felt very caring } \\
\text { towards him so I decided to be } \\
\text { very kind by giving him a } \\
\text { generous portion of my Big Mac } \\
\text { sandwich I had just purchased. } \\
\text { As he ate a portion of my meal }\end{array}$ & $\begin{array}{l}\text { "After a long day at work on a } \\
\text { Friday, I drove to my friends } \\
\text { house to drop off a book that she } \\
\text { loaned me. She wasn't home but } \\
\text { told me she kept a set of her keys } \\
\text { in the mailbox. I let myself in } \\
\text { and dropped the book on her } \\
\text { desk in the office. I grabbed a } \\
\text { clean sheet of paper from the }\end{array}$ \\
\hline
\end{tabular}


greedy and self-serving I was at that stage of my life. It also was disloyal to my employer, which was a nonprofit that gave jobs to poor people. I can't believe what a dishonest person I was back then. I'm so glad that I've changed. I would like to return to the nonprofit one day and atone for what I did." he told me I was very fair towards him. I sure felt good all day after that. I was a little hungry though because he ate more of my meal then I thought he would. I had to go back and get an additional sandwich to fill up my tummy. After that I felt much better and decided to go out and find some more honest people living under bridges that I could give some food to." printer and wrote a short letter to her thanking her for letting me borrow the book. I locked up and returned the keys to the mailbox and drove home. It was another 25 minute drive and by the time I got home I was really beat and just microwaved some left overs and then got into my pajamas and vegged out in front of the television."

\section{Results}

An analysis of variance (ANOVA) showed that the effect of recalling one's negative moral identity on network density was statistically significant, $F(2,137)=3.18, p=.04, \eta \rho^{2}=0.04$. Planned contrasts revealed that individuals who recalled their negative moral identity $(M=0.82$, $S D=0.17)$ triggered a more dense network than those in the control condition $(M=0.73$, $S D=0.23), t(97)=-2.16, p=.03, \mathrm{CI}=[-0.17,-0.01], d=-0.44$, and than those who recalled their positive moral identity $(M=0.73, S D=0.20), t(90)=-2.31, p=.02, \mathrm{CI}=[-0.17,-0.01], d=-0.49$.

\section{Discussion}

Incidentally recalling one's negative moral identity triggered a high-density social network, while recalling one's positive moral identity did not, since participants in that condition had network densities that were no different than the control condition. This finding suggests that the relationship between one's moral identity and cognitive activation of social network is specific to negative moral identity, which poses a threat to one's positive self-concept.

\section{Experiment 4: Self-affirmation Buffers a Threat to Moral Self-concept}

In Experiment 4, we test whether a threat to one's positive self-concept explains the relationship between cheating and high-density network using a mediation-through-moderation approach (Spencer, Zanna, \& Fong, 2005). We manipulated both cheating (whether participants 
likely engaged in it or not) and self-affirmation. We predicted that self-affirmation would moderate the relationship between cheating and network density. Since self-affirmation could reduce one's negative feelings associated with a threat to positive self-concept by sustaining a person's sense of moral adequacy (Steele, 1988), we expected that participants who are affirmed would not trigger high-density networks only in the likely-cheating condition and that those who are not affirmed would trigger high-density networks. On the other hand, self-affirmation is not expected to reduce network density among participants in the no-cheating condition, as their selfconcept has not been threatened by engaging in dishonest behavior.

\section{Method}

Participants. We targeted recruiting 160 participants who passed the attention check at the beginning of the survey, such that the study has $80 \%$ power to detect an effect with a medium-sized effect $\left(\mathrm{f}^{2}=0.15\right)$. One hundred sixty individuals $\left(M_{\text {age }}=33.15, S D_{\text {age }}=10.98 ; 58 \%\right.$ male) participated in a 20-minute online survey through Amazon's Mechanical Turk and received $\$ 0.50$ as well as a bonus payment of up to $\$ 0.90$ based on their outcomes on a series of short tasks.

Procedure. Participants first read that they would be playing an online game and that they would receive a bonus payment based on the outcome of the game. We randomly assigned participants into one of four conditions in a 2 (likely cheating [Opaque] vs. no cheating [Transparent] X 2 (self-affirmation vs. no self-affirmation) between-subjects design

Self-affirmation manipulation. For the manipulation of self-affirmation vs. no selfaffirmation, we gave participants a list of nine personal values and characteristics that people may consider to be important to them (Cohen, Aronson, \& Steele, 2000). Participants in the selfaffirmation condition were told to choose one or two values that they consider most important to 
them, write a paragraph about why this value(s) is important to them personally, and give an example of a time when the value(s) was particularly important in their lives. Participants in the no-affirmation condition were told to choose one or two values from the list that they considered to be least important to them and to write about why these values might be important to someone else.

Cheating manipulation. For the manipulation of likely-cheating vs. no-cheating, we used a die-throwing game adapted from Jiang (2013), in which participants throw a virtual online six-sided die 10 times to earn points that could be converted into real bonus payments. Using a picture of a virtual die, we reminded participants that the pairs of numbers on opposite sides of the die must add up to seven. In each round, the number of points that participants scored depended on the throw of the die (randomly ranging from 1 to 6 ), and on the side (either the Upside [U] or the Downside [D]) that they had chosen before each throw. The visible side of the die, facing up, was called "U," and the opposite side, facing down, was called "D." If a participant chose "D" and rolled a five, then she would earn two points for that throw, whereas if she chose "U," she would receive five points (See Appendix C for the example provided to participants). Each point was translated into three cents, and participants could receive up to \$0.90 after five rounds.

Participants in the opaque condition were asked to choose a side of the die ("U" or "D") in their minds prior to each throw. In each round, after throwing the virtual die, they were asked to indicate the side they had chosen before making the throw to determine their points. Because participants in this condition could change their minds and chose the side that corresponds to the maximum points, this experimental condition allowed cheating. By contrast, participants in the 
transparent condition were asked to choose a side of the die and report it before each throw, so they were not able to change their minds later.

Dependent measure. As in Experiment 1-3, we then used the name generator and demographic questionnaire.

\section{Results}

As a manipulation check, we tested whether individuals in the likely-cheating condition reported higher numbers than those in the no-cheating condition by potentially engaging in dishonest behavior. Indeed, those in the likely-cheating condition $(M=\$ 0.65, S D=0.10)$ earned significantly more than those in the no-cheating condition $(M=\$ 0.54, S D=0.10), t(173)=6.75$, $p<.001, \mathrm{CI}=[-0.14,-0.07], d=-1.04$.

Using a multiple regression model, we found a significant main effect of being tempted to cheat versus no cheating on network density, $\mathrm{B}=0.09, \mathrm{SE}=0.04, p=.02$, and a marginally significant interaction between cheating and self-affirmation, $\mathrm{B}=-0.10, \mathrm{SE}=0.05, p=.06$. A simple slope analysis supports our mediation hypothesis (see Figure 1). When participants were not affirmed with core values, giving them an opportunity to cheat (likely-cheating) predicted activating more dense network, $\mathrm{B}=0.14, \mathrm{SE}=0.06, p=.02$. On the other hand, when participants were affirmed with core values, this no longer significantly predicted the activation of more dense networks, $\mathrm{B}=0.04, \mathrm{SE}=0.03, p=.15$.

Figure 1. Network density across conditions, Experiment 4 


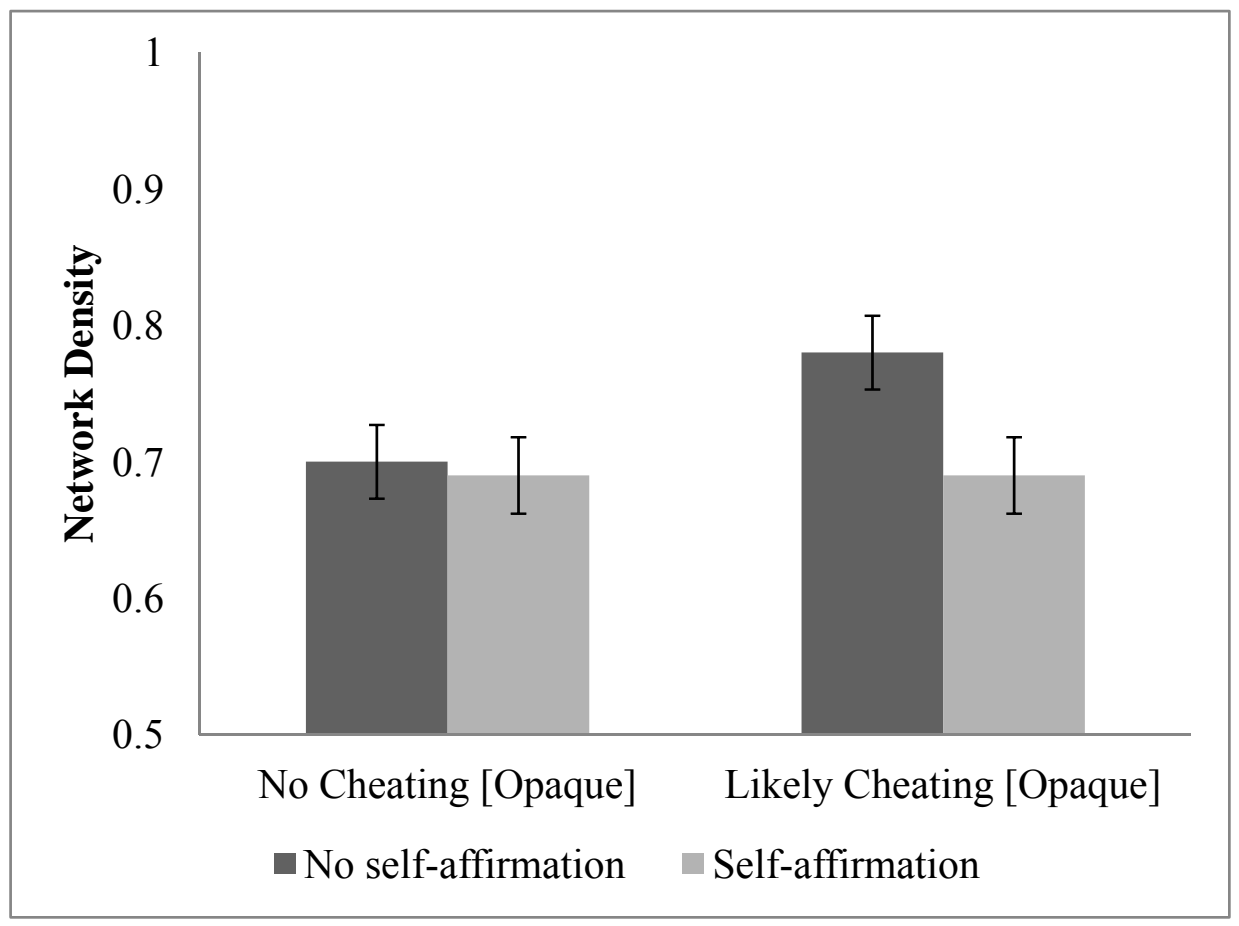

\section{Discussion}

Using a mediation-through-moderation approach, we found that a threat to one's positive self-concept explains the relationship between cheating and high-density network

\section{Experiment 5: The Role of High-Density Networks in Perpetuating Dishonest Behavior}

In Experiment 5, we tested whether triggering a high-density network as a result of dishonest behavior allows individuals to engage in further dishonest behavior. This study used four supposedly unrelated tasks: the virtual die-throwing game from Experiment 4 as a cheating manipulation (Jiang, 2013), a name-generator task as in Experiment 1-4, a four-minute filler task, and the puzzle Boggle to measure dishonesty in the subsequent task (adapted from Marsh \& Bower, 1993). We predicted that triggering a high-density network as a response to being in the likely-cheating condition would predict more subsequent dishonesty and that density would not predict subsequent dishonesty in the no-cheating condition.

\section{Method}


Participants and task. Similar to Experiment 4, we planned to recruit 160 individuals and stopped data collection when we had a total of 160 individuals $\left(M_{\text {age }}=35.42, S D_{\text {age }}=10.88\right.$; $64 \%$ male). They participated in a 25-minute online survey through Amazon's Mechanical Turk and received $\$ 0.40$ as well as a bonus payment of up to $\$ 2.20$ based on the outcomes of a series of short tasks (a maximum of $\$ 1.20$ from the die-throwing game and a maximum of $\$ 1.00$ from the Boggle task).

Subsequent dishonest behavior. We instructed participants to find as many four-letter words as they could from a letter matrix (see Figure 2 for an example) and told them that they would be paid $\$ 0.10$ for each correctly identified word. We also asked participants to follow three rules when constructing their four-letter English words: (1) do not re-use letters in the matrix, (2) all letters must be adjacent, and (3) no proper names allowed. To help participants count the number of correctly identified words, we encouraged them to write down the words they found on a piece of paper. They were given 60 seconds to solve the matrix. They then reported how many they had solved and wrote down the actual words on a separate page for verification. We counted the number of illegitimate words that participants reported that violated the rules of the game: words consisting of more or less than four letters, words that could not be created using our three specified rules, and words that cannot be found in an English dictionary.

Figure 2. Example of nine-letter matrix and depiction of the rules, Experiment 5. 
Correct - REIN

\begin{tabular}{|c|c|c|}
\hline$N$ & $N$ & $\mathrm{I}$ \\
\hline $\mathrm{S}$ & $\mathrm{U}$ & $\mathrm{E}$ \\
\hline $\mathrm{T}$ & $\mathrm{T}$ & $\mathrm{R}$ \\
\hline
\end{tabular}

Incorrect - REST

Letters Must be Adjecent

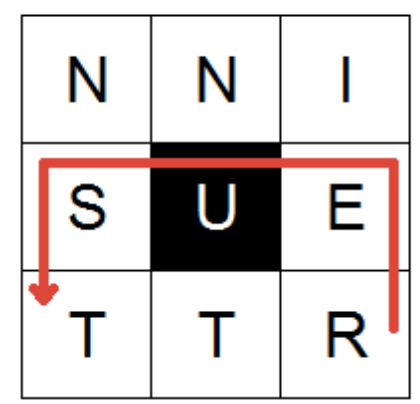

Correct - TUNE

Corners are OK

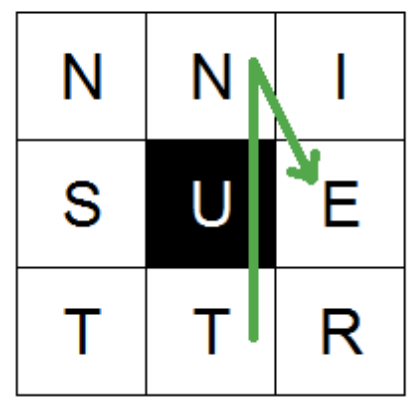

\section{Results}

Similar to Experiment 4, our manipulation check confirmed that those in the likelycheating condition ( $M=\$ 0.62, S D=0.10)$ earned significantly more than those in the no-cheating condition $(M=\$ 0.54, S D=0.10), t(160)=-4.86, p<.001, \mathrm{CI}=[-0.11,-0.05], d=-0.77$.

Replicating the results in Experiment 4, individuals in the likely-cheating condition ( $M=0.74$, $S D=0.21)$ triggered a more dense network than those in the no-cheating condition $(M=0.68$, $S D=0.17), t(158)=-2.00, p=.047, \mathrm{CI}=[-0.12,-0.00], d=-0.32$. However, they did not differ significantly in the number of illegitimate words, $t(158)=-0.60, p=.55, \mathrm{CI}=[-0.59,0.31], d=-$ 0.10 .

Using a negative binomial regression model to account for over-dispersion of the count variable, we found a significant interaction between cheating and self-affirmation, $\mathrm{B}=2.95$, $\mathrm{SE}=1.42, p=.038$ (see Figure 3 for an illustration of the interaction). As predicted, network density predicted more rule-breaking in the Boggle puzzle for participants in the likely-cheating condition, $\mathrm{B}=2.40, \mathrm{SE}=1.00, p=.016$. For those in the no-cheating condition, however, network density did not predict rule breaking, $\mathrm{B}=-0.55, \mathrm{SE}=1.02, p=.59$. This interaction remained robust even when accounting for the likely amount of cheating in the die-rolling task, $\mathrm{B}=3.09$, $\mathrm{SE}=1.46, p=.034$. 
Figure 3. Illustration of the network density by cheating interaction, Experiment 5

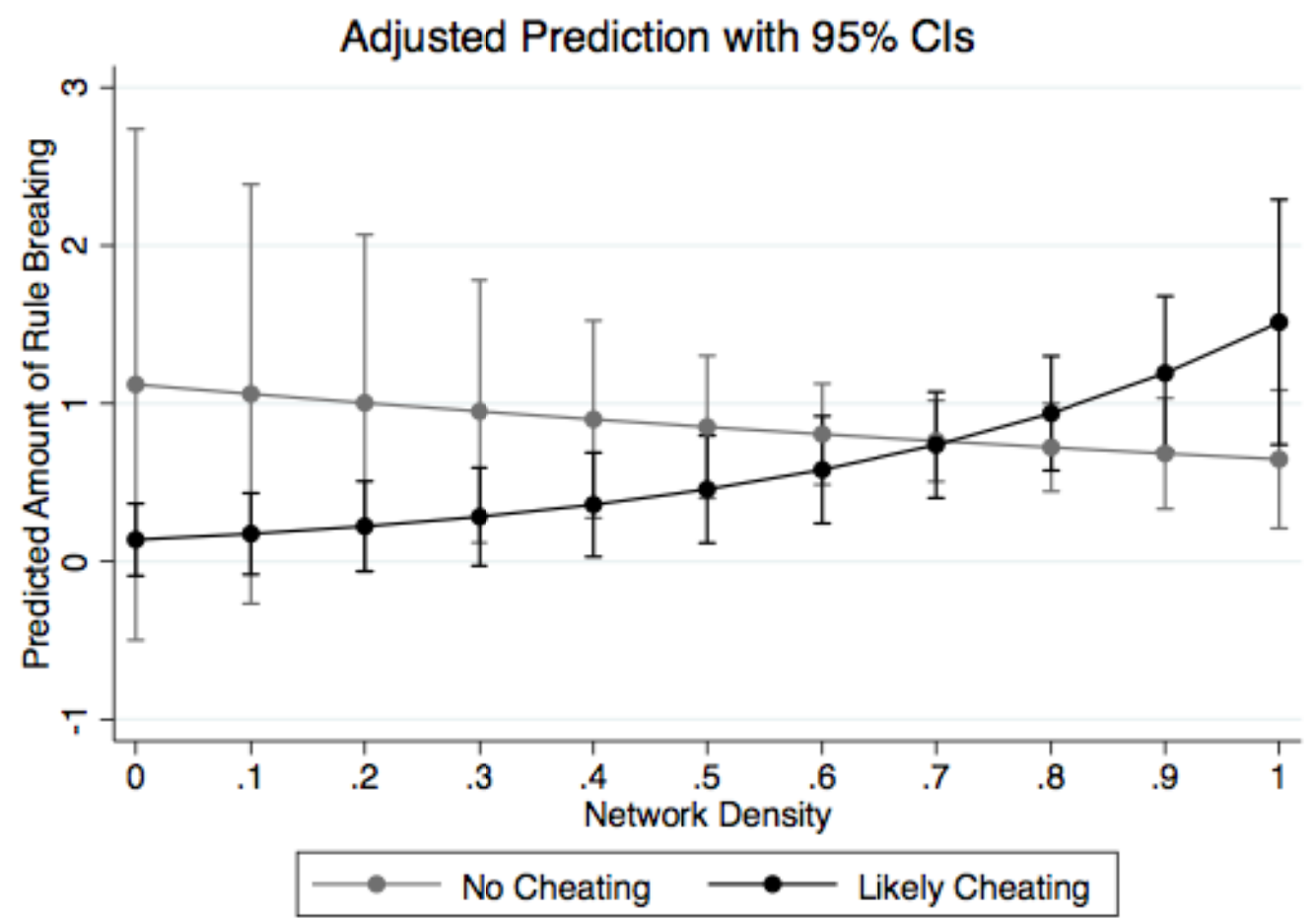

\section{Discussion}

This finding suggests that triggering high-density network in the aftermath of cheating indeed encouraged more subsequent dishonesty.

\section{General Discussion}

The financial consequences of dishonest behavior are both pervasive and evident. According to the Association of Certified Fraud Examiners' 2014 report, employee dishonesty accounted for an estimated global loss of \$3.7 trillion annually (Association of Certified Fraud Examiners, 2014). While an increasing volume of research has sought to delineate the drivers of unethical behavior, the relational and psychological consequences of such behavior have remained relatively understudied. Our work demonstrates that because dishonest behavior poses a threat to one's moral self-concept that can be restored by high-density networks, people think 
about a high-density network to which they belong after behaving dishonestly. Our findings advance the literature on the role of self-concept embedded in social contexts in ethical behavior (Monin \& Jordan, 2009). Just as a threat to self-concept in one domain (e.g., being a sucker) triggers moralization of one's behavior as a means of ego-protection (A. H. Jordan \& Monin, 2008), our results show that a threat to moral self-concept can make a high-density social network readily available.

Our research complicates the idea that morality emerges from social integration and cohesion, which in turn produces cultural expectations and moral practices that promote virtuous behavior (Durkheim, 1912). It is possible that network density could provide an opportunity for moral self-regulation of future behavior. That is, dense and cohesive networks may constrain one's unethical behavior in the future, due to a high level of surveillance and monitoring within the network, and heightened risk of reputational loss when dishonesty is detected (Brass et al., 1998; Burt \& Knez, 1995). However, our findings shed new light on the opposite effect: how one's initial dishonest behavior can create a vicious cycle of future dishonesty by triggering a high-density network as a coping mechanism to reduce a threat to one's moral self-concept. Although recalling a dense network alone did not increase the future dishonest act, it predicted more rule-breaking when the cause of triggering a dense network is related to the discomfort experienced as a result of initial cheating. Although having a cohesive social network can regulate one's moral behavior through shared norms (Coleman, 1988; Schafer, 2014), our work demonstrates that people often construct their own egocentric social network as a way to defend themselves from threatening information.

In sum, the research shows how one's perceived social relationships are central to regulating human morality. At a speculative level, our research raises the possibility that a threat 
to moral self-concept triggered by dishonest behavior may play a role in people's social

motivation to belong to a cohesive network, which may further perpetuate their dishonest

behavior in the future.

\section{References}

Aronson, E. (1968). Dissonance theory: Progress and problems. In R. P. Abelson, E. Aronson, W. J. Mcguire, T. M. Newcomb, M. J. Rosenberg, \& P. H. Tannenbaum, Theories of Cognitive Consistency: A Sourcebook (pp. 5-27). Chicago: Rand McNally. doi: $10.1234 / 12345678$

Aronson, E. (1969). The Theory of Cognitive Dissonance: A Current Perspective. Advances in Experimental Social Psychology (Vol. 4, pp. 1-34). doi:10.1016/s0065-2601(08)60075-1

Association of Certified Fraud Examiners. (2014). Report to the Nations on Occupational Fraud and Abuse: 2014 Global Fraud Study.

Bandura, A. (1990). Selective activation and disengagement of moral control. Journal of Social Issues, 46(1), 27-46. doi:10.1111/j.1540-4560.1990.tb00270.x

Barkan, R. (2007). Measuring Cheating with a Multi-method Scale. Unpublished manuscript.

Barnes, J. A. (1969). Graph theory and social networks: A technical comment on connectedness and connectivity. Sociology, 3(2), 215-232. doi:10.1177/003803856900300205

Blau, P. M. (1977). A macrosociological theory of social structure. American Journal of Sociology. doi:10.2307/2777762

Bowers, C. A., Weaver, J. L., \& Morgan, B. B. (1996). Moderating the performance effects of stressors. Stress and Human Performance, 163-195.

Brass, D. J., Butterfield, K. D., \& Skaggs, B. C. (1998). Relationships and unethical behavior: A social network perspective. Academy of Management Review, 23(1), 14-31. doi:10.5465/amr.1998.192955

Burt, R. S. (1984). Network items and the general social survey. Social Networks, 6(4), 293-339. doi:10.1016/0378-8733(84)90007-8

Burt, R. S., \& Knez, M. (1995). Kinds of third-party effects on trust. Rationality and Society, 7(3), 255-292. doi:10.1177/1043463195007003003

Carlsmith, J. M., \& Gross, A. E. (1969). Some effects of guilt on compliance. Journal of Personality and Social Psychology, 11(3), 232-239. doi:10.1037/h0027039

Chua, V., Madej, J., \& Wellman, B. (2011). Personal communities: the world according to me. The SAGE Handbook of Social Network Analysis, 101-116.

Cialdini, R. B., Darby, B. L., \& Vincent, J. E. (1973). Transgression and altruism: A case for hedonism. Journal of Experimental Social Psychology, 9(6), 502-516. doi:10.1016/00221031(73)90031-0

Cohen, G. L., Aronson, J., \& Steele, C. M. (2000). When beliefs yield to evidence: Reducing biased evaluation by affirming the self. Personality and Social Psychology Bulletin, 26(9), 1151-1164. doi:10.1177/01461672002611011

Coleman, J. S. (1988). Social capital in the creation of human capital. American Journal of Sociology, 94(s1), S95. doi:10.1086/228943

Durkheim, E. (1912). The Elementary Forms of the Religious Life. 
Effron, D. A., \& Knowles, E. D. (2015). Entitativity and intergroup bias: How belonging to a cohesive group allows people to express their prejudices. Journal of Personality and Social Psychology, 108(2), 234-253. doi:10.1037/pspa0000020

Gino, F., Norton, M. I., \& Ariely, D. (2010). The counterfeit self: The deceptive costs of faking it. Psychological Science, 21(5), 712-720. doi:10.1177/0956797610366545

Gneezy, U. (2005). Deception: The Role of Consequences. American Economic Review, 95(1), 384-394. doi:10.1257/0002828053828662

Higgins, E. T. (1987). Self-discrepancy: A theory relating self and affect. Psychological Review, 94(3), 319-340. doi:10.1037/0033-295X.94.3.319

Jiang, T. (2013). Cheating in mind games: The subtlety of rules matters. Journal of Economic Behavior \& Organization.

Jordan, A. H., \& Monin, B. (2008). From sucker to saint moralization in response to self-threat. Psychological Science, 19(8), 809-815. doi:10.1111/j.1467-9280.2008.02161.x

Jordan, J., Mullen, E., \& Murnighan, J. K. (2011). Striving for the moral self: The effects of recalling past moral actions on future moral behavior. Personality and Social Psychology Bulletin, 37(5), 701-713. doi:10.1177/0146167211400208

Klass, E. T. (1978). Psychological effects of immoral actions: The experimental evidence. Psychological Bulletin, 85(4), 756-771. doi:10.1037/0033-2909.85.4.756

Labianca, G., Brass, D. J., \& Gray, B. (1998). Social Networks and Perceptions of Intergroup Conflict: The Role of Negative Relationships and Third Parties. Academy of Management Journal, 41(1), 55-67. doi:10.2307/256897

Marsden, P. V. (1987). Core Discussion Networks of Americans. American Sociological Review, 52(1), 122. doi:10.2307/2095397

Marsh, R. L., \& Bower, G. H. (1993). Eliciting cryptomnesia: Unconscious plagiarism in a puzzle task. Journal of Experimental Psychology: Learning, Memory, and Cognition, 19(3), 673-688. doi:10.1037/0278-7393.19.3.673

Mazar, N., Amir, O., \& Ariely, D. (2008). The dishonesty of honest people: A theory of selfconcept maintenance. Journal of Marketing Research, 45(6), 633-644.

McCornack, S. A., \& Levine, T. R. (1990). When lies are uncovered: Emotional and relational outcomes of discovered deception. Communication Monographs, 57(2), 119-138. doi:10.1080/03637759009376190

McMillen, D. L. (1971). Transgression, self-image, and compliant behavior. Journal of Personality and Social Psychology, 20(2), 176-179. doi:10.1037/h0031686

McMillen, D. L., \& Austin, J. B. (1971). Effect of positive feedback on compliance following transgression. Psychonomic Science, 24(2), 59-61. doi:10.3758/BF03337892

McPherson, M., Smith-Lovin, L., \& Brashears, M. E. (2009). Models and Marginals: Using Survey Evidence to Study Social Networks. American Sociological Review, 74(4), 670-681. doi:10.1177/000312240907400409

Miller, G. R., Mongeau, P. A., \& Sleight, C. (1986). Invited Article Fudging with Friends and Lying to Lovers: Deceptive Communication in Personal Relationships. Journal of Social and Personal Relationships, 3(4), 495-512. doi:10.1177/0265407586034006

Monin, B., \& Jordan, A. H. (2009). The Dynamic Moral Self: A Social Psychological

Perspective. (D. Narvaez \& D. K. Lapsley)Explorations in Moral Psychology (pp. 341-354). Cambridge: Cambridge University Press. doi:10.1017/cbo9780511627125.016

Reagans, R., \& McEvily, B. (2003). Network structure and knowledge transfer: The effects of cohesion and range. Administrative Science Quarterly, 48(2), 240. doi:10.2307/3556658 
Reed, A., Aquino, K., \& Levy, E. (2007). Moral identity and judgments of charitable behaviors. Journal of Marketing, 71(1), 178-193. doi:10.1509/jmkg.71.1.178

Sachdeva, S., Iliev, R., \& Medin, D. L. (2009). Sinning saints and saintly sinners the paradox of moral self-regulation. Psychological Science, 20(4), 523-528. doi:10.1111/j.14679280.2009.02326.x

Sagarin, B. J., L Rhoads, von, K., \& Cialdini, R. B. (1998). Deceiver's distrust: Denigration as a consequence of undiscovered deception. Personality and Social Psychology Bulletin, 24(11), 1167-1176. doi:10.1177/01461672982411004

Schafer, M. H. (2014). Schema via structure? Personal network density and the moral evaluation of infidelity. Sociological Forum, 29(1), 120-136. doi:10.1111/socf.12072

Scott, J. (1991). Networks of corporate power: A comparative assessment. Annual Review of Sociology. doi:10.2307/2083340

Shaw, M. E., \& Shaw, L. M. (1962). Some effects of sociometric grouping upon learning in a second grade classroom. The Journal of Social Psychology, 57(2), 453-458. doi:10.1080/00224545.1962.9710941

Shu, L. L., \& Gino, F. (2012). Sweeping dishonesty under the rug: How unethical actions lead to forgetting of moral rules. Journal of Personality and Social Psychology, 102(6), 1164-1177. doi: $10.1037 / \mathrm{a} 0028381$

Shu, L. L., Gino, F., \& Bazerman, M. H. (2011). Dishonest deed, clear conscience: When cheating leads to moral disengagement and motivated forgetting. Personality and Social Psychology Bulletin, 37(3), 330-349. doi:10.1177/0146167211398138

Small, M. L., Deeds Pamphile, V., \& McMahan, P. (2015). How stable is the core discussion network? Social Networks, 40, 90-102. doi:10.1016/j.socnet.2014.09.001

Spencer, S. J., Zanna, M. P., \& Fong, G. T. (2005). Establishing a causal chain: Why experiments are often more effective than mediational analyses in examining psychological processes. Journal of Personality and Social Psychology, 89(6), 845-851. doi:10.1037/00223514.89.6.845

Steele, C. M. (1988). The psychology of self-affirmation: Sustaining the integrity of the self. Advances in Experimental Social Psychology. doi:10.1016/S0065-2601(08)60229-4

Stokes, J. P. (1985). The relation of social network and individual difference variables to loneliness. Journal of Personality and Social Psychology, 48(4), 981-990. doi:10.1037/0022-3514.48.4.981

Türker, D., \& Altuntaş, C. (2014). Analysis of unethical behaviors in social networks: An application in the medical sector. Procedia-Social and Behavioral Sciences, 150, $1177-$ 1186. doi:10.1016/j.sbspro.2014.09.133 


\section{Supplementary Information Appendix:}

Thick as Thieves?

Dishonest Behavior and Egocentric Social Networks

Jooa Julia Lee, ${ }^{1}$ Dong-Kyun Im, ${ }^{2}$ Bidhan Parmar, ${ }^{3}$ Francesca Gino ${ }^{1}$

${ }^{1}$ Harvard University, Cambridge, MA 02138, USA

${ }^{2}$ University of Seoul, South Korea

${ }^{3}$ University of Virginia, Charlottesville, VA, 22906, USA 


\section{Appendix A.}

Scale to measure the frequency of ethically questionable behavior (Experiment 1).

Q1. How frequently do you engage in the following behaviors? ( 1 = "never", 5 = "all of the time, 6 = "not applicable")

- Taking home office supplies from work.

- Boarding a plane before your number is called.

- Lying to an insurance company about the value of goods that were damaged.

- Telling your supervisor that progress has been made on a project when none has been made at all.

- Inflating your business expense report.

- Buying a garment, wearing it, and returning it.

- Being in the express line with too many groceries.

Q2. How frequently do you say the following lies? $(1=$ "never", 5 = "all of the time, 6 = "not applicable")

- Yes, John was with me last night.

- It was good meeting you. Let’s have lunch sometime.

- Sure, I will start working on that tonight.

- My GPA is 4.0.

- I thought I already sent that email out. I am sure I did.

- Sorry I am late, traffic was terrible. 


\section{Appendix B.}

\section{Self-affirmation manipulation (Experiment 4).}

Q1. Please choose 2-3 values that are most [least] important to you. Although several of the values might be [not] important to you, please only select 2-3 values.

- Being intelligent

- Being civic-minded

- Being creative

- Relationships with friends

- Relationships with family

- Being independent

- Being honest

- Being healthy

- Being kind

- Being unique

- Sense of humor

- Being religious

- Living in the moment

- Being good-looking

- Being athletic

Q2. You have chosen the following values as most important to you. Please think about times when these values were important to you, and describe in a few sentences why your selected values were important to you. 


\section{Appendix C.}

\section{Cheating manipulation (Experiment 4-5).}

Please read the following instructions very carefully.

In this game, you will be asked to roll a six-sided die five times.

In each round, the number of points that you score depends on the throw of the die as well as the side that you have chosen in that round. Each round consists of one throw. Before throwing, you have to choose the relevant side for that round. Please note that the pairs of numbers on opposite sides of the die add up to 7: 1 vs. 6, 2 vs. 5, and 3 vs. 4, and vice versa. In the game, the visible side facing up of the dice is "the up side" (i.e., "U"), and the opposite, invisible side facing down is "the down side" (i.e., "D"). Note that the die outcomes are random, and the outcome you see on the screen corresponds to the upside.

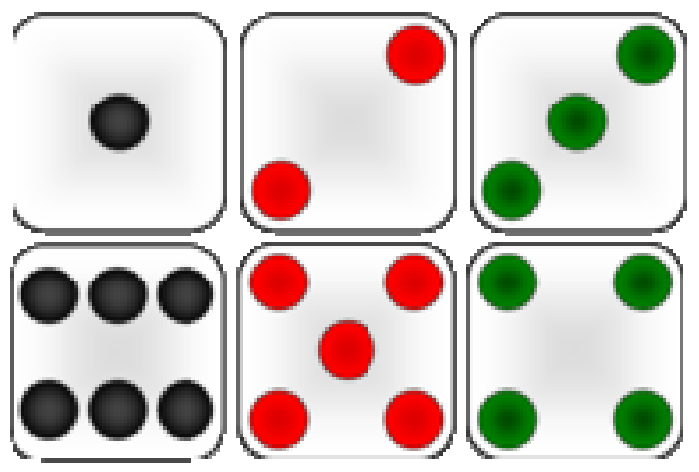

For instance, if you have chosen "D" and the die outcome turns up to be "4", you will earn 3 points for that throw, whereas if you have chosen "U" in your mind, you earn 4 points. Across the 5 rounds, you can earn a maximum of 30 points (minimum of 10 points). Each point is worth 3 cent, so you can make between $\$ 0.30$ and $\$ 0.90$.

We are going to ask you a question to test if you understood the rule of the game. Unfortunately, if you do not get these follow-up questions right, you cannot participate in this study. Please press NEXT when you're ready to take a quiz and play!

Eligibility Questions (only those who answer the two questions correctly were able to participate in this study):

Q1. If you have chosen " $U$ " and the die outcome turns up to be 3, how many points will you earn?

Q2. If you have chosen "D" and the die outcome turns up to be 2, how many points will you earn? 